\title{
Monte Carlo modelling of imperfections in two-dimensional photonic crystals ${ }^{1}$
}

\author{
Tim Langtry ${ }^{\mathrm{a}}$ Lindsay Botten ${ }^{\mathrm{a}}$ Ara Asatryan ${ }^{\mathrm{b}}$ \\ Ross McPhedran ${ }^{b}$

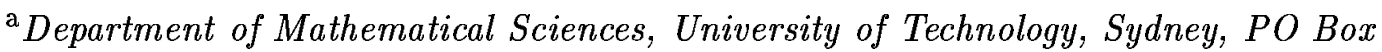 \\ 123, Broadway NSW 2007, Australia \\ ${ }^{\mathrm{b}}$ School of Physics A28, University of Sydney, NSW 2006, Australia
}

\begin{abstract}
In this paper we describe a Monte Carlo simulation of imperfections in photonic crystals, a new class of materials with optical properties that offer promise in a range of potential applications in the areas of information and communications technology. We describe the relevant physical and structural properties of these materials and outline the derivation of a theoretical model. We then present a Monte Carlo investigation of the tolerance of these materials to fabrication defects.
\end{abstract}

\section{Introduction}

In recent years there has been much interest in photonic crystals, a new class of materials with optical properties that offer promise in a range of potential applications in communications technology. Photonic crystals of dimension $s$ are materials in which the refractive index varies periodically with position along $s$ axes. Examples of one-dimensional crystals include multilayer thin films (see for example [1]), which have been widely studied - applications include dielectric mirrors and optical filters. Recently, higher-dimensional photonic crystals have been intensively studied [2-4]. Such materials may be constructed by fabricating a material matrix with inclusions that are centred at points of a

Email addresses: Tim.Langtry@uts .edu.au (Tim Langtry), Lindsay. Botten@uts.edu. au (Lindsay Botten), ara@physics.usyd.edu .au (Ara Asatryan), r.mcphedran@physics.usyd.edu.au (Ross McPhedran).

1 The authors wish to acknowledge the support of the Australian Research Council, the Australian Centre for Advanced Computing and Communications (ac3) and the Australian Partnership for Advanced Computing (APAC) National Facility. 
two- or three-dimensional lattice. The examples used in this paper are based on a square lattice with lattice constant $d$. In particular, when the period of the refractive index is of an order comparable with the wavelength of an incident electromagnetic field, interferometric action can yield ranges of frequencies and wave vectors for which the fields cannot propagate through the material (photonic band gaps) - the local density of states (LDOS), a useful indicator of electromagnetic field confinement, diminishes exponentially, on average, with the depth of penetration into the structure [5].

The introduction of defects into a photonic crystal's lattice structure can allow local propagation into the structure of an electromagnetic wave with a frequency belonging to a band gap: a localised mode analogous to the impurity states of semiconductors. These defects change the radiation dynamics of the crystal and, by controlling the nature and location of such defects, it is possible to control the direction of propagation on a scale comparable with the wavelength - in effect allowing the crystal to mould the flow of light in various ways. It is this property that gives hope of many significant applications, including the fabrication of microscopic lasers, new families of optical fibres, and the fabrication of optical waveguides and switches which may be used as components in future integrated photonic circuits [2,3]. Consequently, it is of interest to determine the sensitivity of a crystal's radiation dynamics to imperfections in its geometric and material properties.

Much of the research to date has been devoted to development of methods to compute transmission spectra and the related band diagrams that characterise the frequencies and directions for which light may propagate within a crystal [4]. These methods, however, provide little insight into a crystal's radiation dynamics, which are characterised by the local density of states (LDOS), studied recently in [5-7]. The LDOS at a given point determines the density of available modes of the structure at the point. In turn, this determines the efficiency of the radiation of an embedded source at this position. For more details see [5-7] and the references cited therein. In this paper we describe the application of Monte Carlo simulation to the modelling of structural defects in two-dimensional photonic crystals. In $\S 2$ we give a brief description of a Rayleigh multipole method for calculating the relevant Green's function and LDOS. The implementation of this method and its application to the study of localised modes is described in $\S 3$, and in $\S 4$ we present a Monte Carlo simulation study based on the method.

\section{Green's function and LDOS for a $2 \mathrm{D}$ crystal-TM polarisation}

We consider a two-dimensional crystal comprising a finite cluster of $N_{c}$ circular cylinders of infinite length aligned with the $z$ axis, with cylinder $l$ centred 

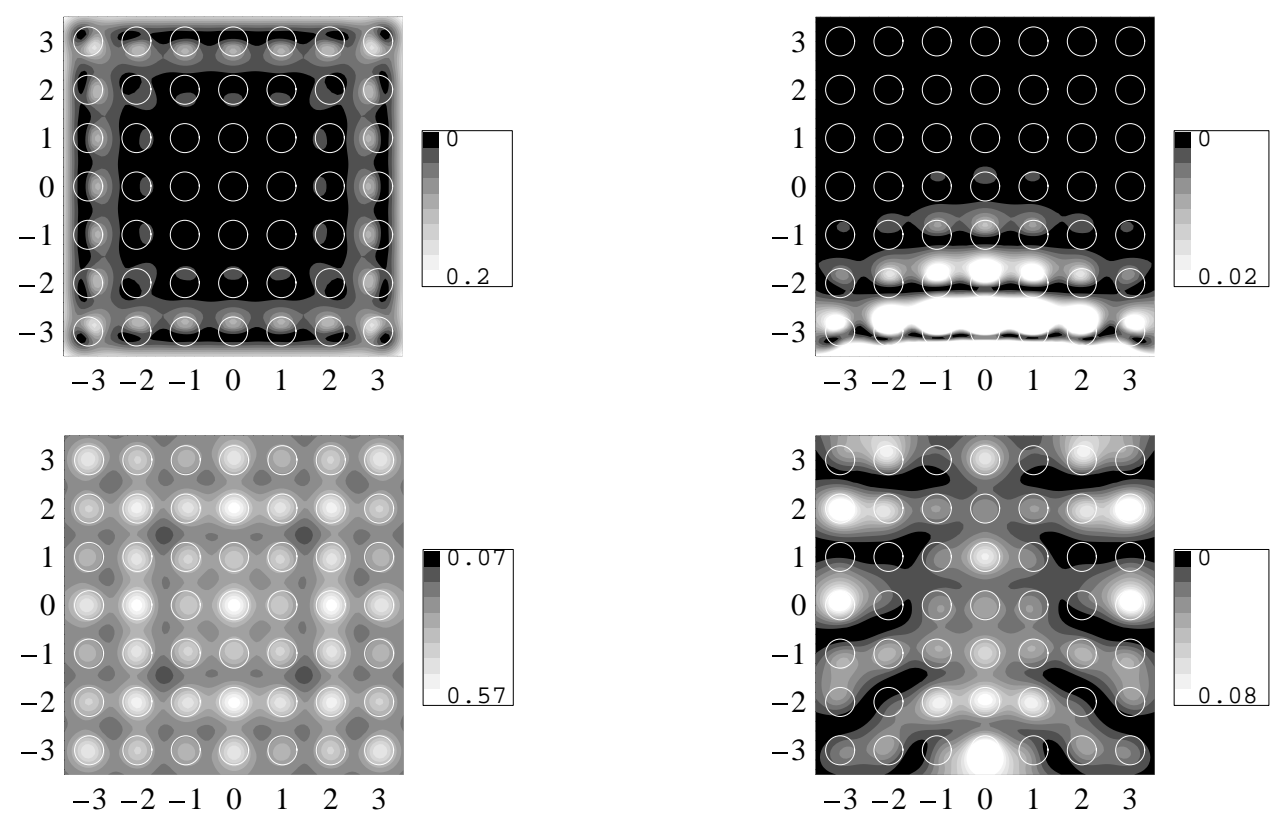

Fig. 1. Contour plots in the $x, y$ plane of LDOS $(\rho)$ (left) and square modulus of Green's function $\left(\left|V_{e}\right|^{2}\right)$ (right) for TM polarisation for a square lattice $\left(N_{c}=49\right)$ of infinite circular cylinders, whose positions are indicated by circles. The crystal is regular (no defects), with cylinder radii $0.3 d$ and refractive index 3.0. The field plots correspond to normalised wavelengths of $\lambda / d=3.3$ (top), in the band gap, and $\lambda / d=5.5$. (bottom), a pass band.

at $\mathbf{c}_{l}$ and having radius $a_{l}$ and refractive index $n_{l}$. The response of such a crystal to a cylindrical wave emanating from a line source at $\mathbf{c}_{s}$ in the $x, y$ plane and parallel to the cylinder axes is given by the two-dimensional Green's function for the source point. More generally, the radiation dynamics at a particular point of a photonic crystal are characterised by the LDOS. For a given frequency the LDOS provides the spectral distribution of modes to which a fluorescent line source can couple. For large LDOS values, the probability of emission at that frequency is enhanced; correspondingly, a small LDOS value indicates a lower probability of emission. In a band gap of an infinite crystal, the LDOS is zero: emission cannot occur. The LDOS has previously been calculated in infinite periodic structures of one dimension, and at isolated points within the unit cell of a three-dimensional lattice of spheres $[8,9]$. In a band gap of a finite crystal the LDOS is small, but not zero-it is thus a parameter of physical significance for applications. Accordingly, there is considerable interest in the development of efficient methods for computing densities of states, and related measures, for finite crystals of realistic size. Recent papers [5-7] describe such a method for two-dimensional structures, which we outline briefly below.

Figure 1 presents greyscale contour plots in the $x, y$ plane (a plane of symmetry) of both the Green's function for a line source at $(0,-4)$ and the LDOS, 
both for a pass band and for a wavelength in a band gap. In the band gap case $(\lambda / d=3.3)$, high values of the Green's function for $y<-2$ together with low values for $y>-2$ indicate strong reflection of the incident field by the structure. The LDOS plot for this wavelength indicates a low field strength within the crystal but a relatively high field strength at the surface layer. For the wavelength in the pass band $(\lambda / d=5.5)$, however, the LDOS plot indicates that propagation within the crystal is allowed, which behaviour is demonstrated by the Green's function plot.

In the following we assume that the crystal material is macroscopic and isotropic, the dielectric parameter is independent of frequency and piecewise constant and magnetic permeability is constant $(=1)$. We also assume sinusoidal time dependence of the electric and magnetic fields, that there are no free charges, and the current is a line source parallel to the $z$ axis with angular frequency $\omega=k_{0} c$. Maxwell's equations then have the form

$$
\begin{array}{cc}
\nabla \times \mathbf{E}+i k_{0} Z_{0} \mathbf{H}=\mathbf{0} & \nabla \times \mathbf{H}-i\left(k_{0} / Z_{0}\right) n^{2}(\mathbf{r}) \mathbf{E}=J \hat{\mathbf{z}} \\
\nabla \cdot \mathbf{H}=\mathbf{0} & \nabla \cdot \mathbf{E}=\mathbf{0}
\end{array}
$$

where $k_{0}$ is the free-space wavenumber, $Z_{0}$ is the impedance of free space, $n(\mathbf{r})$ is the refractive index and $\mathbf{r}$ denotes position in the plane normal to the cylinders. When the wave vector lies in the plane normal to the cylinder axes (in-plane incidence), the field problem decouples into two fundamental polarisations in which either the electric field (TM polarisation) or magnetic field (TE polarisation) aligns with the cylinder axes. We consider only the TM polarisation that is parametrised by the single non-trivial component of the two-dimensional Green's tensor, which we denote by $V^{e}$. In this case Maxwell's equations yield the two-dimensional boundary value problem for $V^{e}$ in which

$$
\nabla^{2} V^{e}\left(\mathbf{r} ; \mathbf{c}_{s}\right)+k_{0}^{2} n^{2}(\mathbf{r}) V^{e}\left(\mathbf{r} ; \mathbf{c}_{s}\right)=\delta\left(\mathbf{r}-\mathbf{c}_{s}\right)
$$

and $V^{e}$ and its normal derivative $\nu \cdot \nabla V^{e}$ are continuous at cylinder interfaces. In the vicinity of each cylinder $l$, we expand the exterior field in local coordinates $\mathbf{r}_{l}=\left(r_{l}, \theta_{l}\right)=\mathbf{r}-\mathbf{c}_{l}$,

$$
V^{e}\left(\mathbf{r} ; \mathbf{c}_{s}\right)=\sum_{m=-\infty}^{\infty}\left[A_{m}^{l} J_{m}\left(k_{0} r_{l}\right)+B_{m}^{l} H_{m}^{(1)}\left(k_{0} r_{l}\right)\right] e^{i m \theta_{l}}
$$

using a multipole expansion involving both irregular components (coefficients $B_{m}^{l}$ ), denoting scattered field sources associated with this cylinder, and regular components $\left(A_{m}^{l}\right)$. This expansion is valid only in the annulus extending from the surface of cylinder $l$ to the surface of the nearest cylinder or source. An expansion valid everywhere in the crystal matrix [7] is given by:

$$
V^{e}\left(\mathbf{r} ; \mathbf{c}_{s}\right)=V_{0}^{e}\left(\mathbf{r} ; \mathbf{c}_{s}\right)+\sum_{q=1}^{N_{c}} \sum_{m=-\infty}^{\infty} B_{m}^{q} H_{m}^{(1)}\left(k_{0}\left|\mathbf{r}-\mathbf{c}_{q}\right|\right) e^{i m \arg \left(\mathbf{r}-\mathbf{c}_{q}\right)}
$$


where $V_{0}^{e}\left(\mathbf{r} ; \mathbf{c}_{s}\right)=\chi^{\text {ext }}\left(\mathbf{c}_{s}\right) H_{0}^{(1)}\left(k_{0}\left|\mathbf{r}-\mathbf{c}_{s}\right|\right) /(4 i)$ is the solution of the boundary value problem in the absence of a cylinder cluster. Here $\chi^{\text {ext }}\left(\mathbf{c}_{s}\right)$, the exterior characteristic function, is 1 for a source exterior to all cylinders and 0 otherwise. Similarly, letting $\chi_{l}^{\text {int }}\left(\mathbf{c}_{s}\right)$ be 1 if the source lies in cylinder $l$ and 0 otherwise, the field expansion inside cylinder $l$ is given by

$$
V^{e}\left(\mathbf{r} ; \mathbf{c}_{s}\right)=\frac{\chi_{l}^{\mathrm{int}}\left(\mathbf{c}_{s}\right)}{4 i} H_{0}^{(1)}\left(k_{0} n_{l}\left|\mathbf{r}-\mathbf{c}_{s}\right|\right)+\sum_{m=-\infty}^{\infty} C_{m}^{l} J_{m}\left(k_{0} n_{l}\left|\mathbf{r}-\mathbf{c}_{l}\right|\right) e^{i m \arg \left(\mathbf{r}-\mathbf{c}_{l}\right)}
$$

Equating the expansions in (1) and (2) yields a field identity of form

$$
\mathbf{A}=\mathbf{S B}+\chi^{\mathrm{ext}} \mathbf{K}
$$

where $\mathbf{S}$ is a partitioned matrix specifying multipole contributions associated with sources on each scatterer, $\mathbf{K}$ is a partitioned vector containing the coefficients of the source at $\mathbf{c}_{s}$ with respect to a local expansion, and $\mathbf{A}, \mathbf{B}$ are partitioned vectors containing the multipole coefficients for all cylinders. Applying the boundary conditions then gives identities $\mathbf{B}=\mathbf{R A}+\mathbf{T} \chi^{\mathrm{int}} \mathbf{Q}$ and $\mathbf{C}=\mathbf{T}^{\prime} \mathbf{A}+\mathbf{R}^{\prime} \chi^{\text {int }} \mathbf{Q}$, where $\mathbf{R}, \mathbf{R}^{\prime}, \mathbf{T} \mathbf{T}^{\prime}$ denote matrices of cylindrical harmonic reflection and transmission coefficients and the term $\mathbf{Q}$ represents a source interior to a cylinder. These, together with (4), yield the Rayleigh identity:

$$
(\mathbf{I}-\mathbf{R S}) \mathbf{B}=\mathbf{R} \chi^{\text {ext }} \mathbf{K}+\mathbf{T} \chi^{\text {int }} \mathbf{Q}
$$

from which $\mathbf{B}$, and hence $\mathbf{A}, \mathbf{C}$ and $V^{e}$, are determined. The LDOS, denoted by $\rho(\mathbf{r} ; \omega)$, is then defined [10] as a function of spatial position $\mathbf{r}$ and temporal angular frequency $\omega$ by

$$
\rho(\mathbf{r} ; \omega)=-\frac{2 \omega}{\pi c^{2}} V^{e}(\mathbf{r} ; \mathbf{r} ; \omega)
$$

We note here that in free space $\rho$ has value 0.25 . Also note that these field constructions are exact up to the truncation of the series in (2) and (3). In implementing the method, the number of cylinders $N_{c}$ and the number of terms retained in these series govern the size of the dense matrix I - RS in (5). In the examples used in this paper, 11 terms $(m=5)$ were used.

\section{Implementation}

As noted in $§ 1$, it is of interest to determine the sensitivity of a crystal's local radiation dynamics to imperfections in the geometric and material properties of the crystal structure that may be introduced during manufacture - in particular, perturbations in the cylinder radii, refractive indices and positions. Here we describe a Monte Carlo simulation of an ensemble of $N_{I}$ photonic 
crystals using a given design containing $N_{c}$ cylinders, which we refer to as the ideal structure, characterised by parameter vectors defining the locations of the cylinder centres, their radii and their refractive indices (which may be complex-valued). Each realisation in the ensemble is formed by the addition to these parameter vectors of corresponding random perturbations: $\epsilon_{\mathbf{x}}, \epsilon_{\mathbf{y}}, \epsilon_{\mathbf{a}}$, $\epsilon_{\mathbf{n}_{r}}, \epsilon_{\mathbf{n}_{i}}$ (the first three representing perturbations of the $x$ and $y$ coordinates of the cylinder centres and the cylinder radii, and the last two representing perturbations of the real and imaginary parts of the refractive indices). The perturbing vectors are assumed to be uniformly distributed on a specified interval of form $\left[-\epsilon_{\mathbf{v}, \max }, \epsilon_{\mathbf{v}, \max }\right]$, where $\mathbf{v}$ is one of $\mathbf{x}, \mathbf{y}, \mathbf{a}, \mathbf{n}_{r}, \mathbf{n}_{i}$.

The values of $\rho$ and $\left|V^{e}\right|^{2}$ for each realisation in the ensemble are computed at each point of a user-specified grid $\mathcal{P}$ in the $x, y$ plane. Let $\epsilon=$ $\left(\epsilon_{\mathbf{x}}, \epsilon_{\mathbf{y}}, \epsilon_{\mathbf{a}}, \epsilon_{\mathbf{n}_{r}}, \epsilon_{\mathbf{n}_{i}}\right)$ and denote by $\rho(\mathbf{r} ; \omega, \epsilon)$ and $\left|V^{e}\right|^{2}(\mathbf{r} ; \omega, \epsilon)$ the values of $\rho(\mathbf{r} ; \omega)$ and $\left|V^{e}\right|^{2}(\mathbf{r} ; \omega)$ for the realisation with perturbation $\epsilon$. Also, denote by $\langle\rho(\mathbf{r} ; \omega)\rangle$ and $\left\langle\left|V^{e}\right|^{2}(\mathbf{r} ; \omega)\right\rangle$ the mean values (over $\epsilon$ ) of $\rho(\mathbf{r} ; \omega, \epsilon)$ and $\left|V^{e}\right|^{2}(\mathbf{r} ; \omega, \epsilon)$ respectively. The simulation objective is to estimate, at the grid points, values of $\langle\rho(\mathbf{r} ; \omega)\rangle$ and $\left\langle\left|V^{e}\right|^{2}(\mathbf{r} ; \omega)\right\rangle$ by corresponding ensemble averages, denoted by $\widehat{\rho}_{\left(N_{I}\right)}(\mathbf{r} ; \omega)$ and $\widehat{\left.V^{e}\right|^{2}}{ }_{\left(N_{I}\right)}(\mathbf{r} ; \omega)$. In practice we also track estimates of $\phi_{\rho}:=\left\|(\langle\rho(\mathbf{r} ; \omega)\rangle)_{\mathbf{r} \in \mathcal{P}}\right\|_{2}$ and $\phi_{V}:=\left\|\left(\left\langle\left|V^{e}\right|^{2}(\mathbf{r} ; \omega)\right\rangle\right)_{\mathbf{r} \in \mathcal{P}}\right\|_{2}$, and the standard errors for these variables (see, for example, [11]). Two methods were used to generate the perturbations in the initial program: Lapack's pseudo-random number generator [12], and the Faure low discrepancy sequence [13]. The first 500 terms in the Faure sequence were skipped in the example described below.

The simulation program is well-suited to a parallel environment and has been implemented in Fortran90 using MPI to support multiple slave processes, each executing the code for a single realisation. A master process is responsible for generating the realisations, distributing these to the slave processes and collating their results. Each slave process itself manifests substantial loop-level parallelism and, when executed in a shared memory environment, the code can use OpenMP to distribute the slave process over multiple processors.

As an example we consider an ideal crystal composed of 49 cylinders of radius 0.3 units and refractive index 3.0, with cylinders centred at the integer points of $[-3,3]^{2}$. Note that the crystal design and its optical properties are scalable, so when specifying the positions of cylinder centres we may use integer lattice points. In the notation of $\S 1$, this is equivalent to choosing $d=1$. An ensemble of 500 realisatons of this design was created with realisations subject to perturbations with $\epsilon_{\mathbf{x}, \max }=0.01, \epsilon_{\mathbf{y}, \max }=0.02, \epsilon_{\mathbf{a}, \max }=0.1, \epsilon_{\mathbf{n}_{r}, \text { max }}=0.2$ and $\epsilon_{\mathbf{n}_{i}, \max }=0.2$. Values of $\widehat{\left.V^{e}\right|^{2}}{ }_{(500)}$ for the TM polarisation were computed on a square grid $\mathcal{P}$ of 1600 points in $[-3.5,3.5]^{2}$. Figure 2 gives the estimated standard error for $\phi_{V}$. Fitting this data to the expression $c N_{I}^{b}$ yields $b=-0.39$ for the Lapack pseudo-random generator, and $b=-0.36$ for the Faure sequence. 

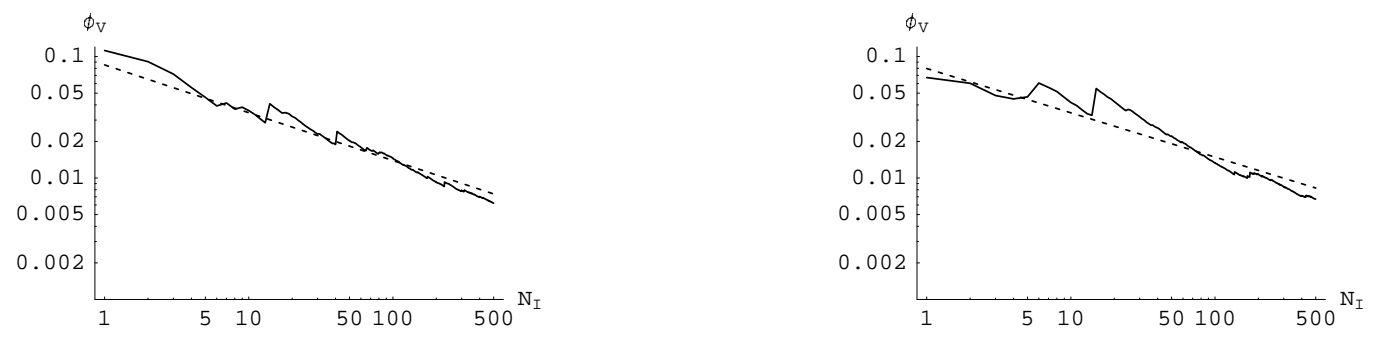

Fig. 2. Plots of estimated standard error for $\phi_{V}$ versus number of realisations $N_{I}$, for a square cluster of 49 circular cylinders of infinite length aligned with the $z$ axis. The cylinders have common refractive index 3.0 and radius $0.3 d$ units, where $d$ is the magnitude of the lattice basis vectors. The results obtained with the Lapack generator are plotted on the left, and those obtained with the Faure sequence on the right. The dashed lines are plots of the fitted functions $f\left(N_{I}\right)=c N_{I}^{b}$.

The value of $\phi_{V}$ obtained after 500 realisations with the Lapack generator was 0.44153 with an estimated standard error of 0.0062 . The Faure sequence yielded $\phi_{V}=0.44922$ with an estimated standard error of 0.0067 .

\section{Application: imperfections in a photonic waveguide}

As described in $\S 1$, the introduction of flaws in the lattice structure of a photonic crystal potentially allows the localised propagation of modes belonging to a band gap of a corresponding flawless crystal. Structuring the flaws to produce a channel through the crystal can then produce a photonic waveguide on a scale comparable with the wavelength of light in the band gap. Such a guide may contain sharp corners around which light is guided by the local radiation dynamics (see Fig. 3). The transmission properties depend critically on the lattice structure of the crystal. Here we consider a Monte Carlo study of transmission for waveguides using the design containing 226 cylinders illustrated in Fig. 3, which we refer to as the ideal structure. The cylinders of the cluster are
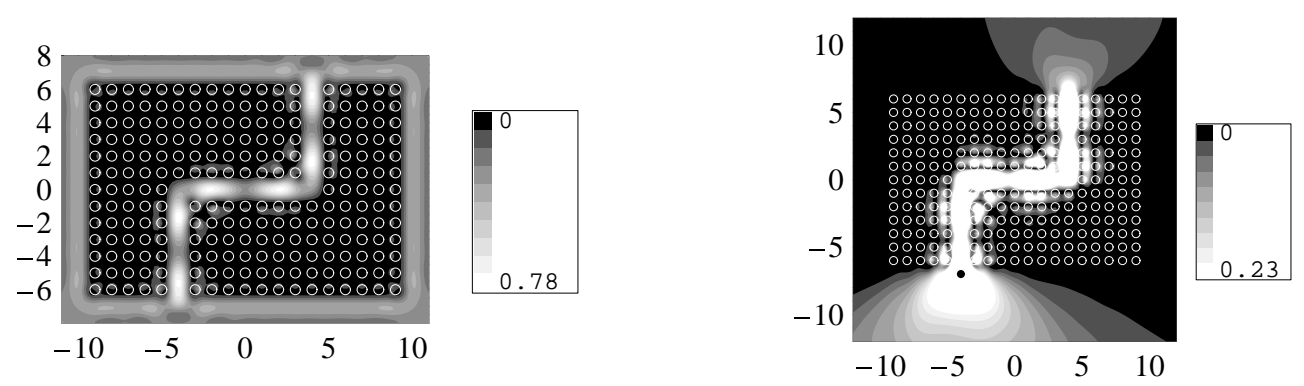

Fig. 3. Contour plots of $\rho$ (left) and $\left|V_{e}\right|^{2}$ (right) for TM polarisation for a waveguide $\left(N_{c}=226\right)$ composed of infinite circular cylinders with no disorder. The cylinders have radius $0.3 d$ and refractive index 3.0. The black dot at $(-4,-7)$ in the second plot indicates the position of a line source. The wavelength used is $3.3 d$. 
Table 1

Values of $\widehat{\rho}_{(50)}$ and $\widehat{\left|V^{e}\right|^{2}}{ }_{(50)}$ at selected points for TM polarisation for the waveguide of Fig. 3 with a source at $(-4,-7)$. The point $(0,0)$ is the centre point of the channel, $(-4,3.5)$ lies in the crystal matrix in line with, but beyond, the first segment of the channel, and $(4,7)$ lies just beyond the channel exit.

\begin{tabular}{l||cc|cc|cc}
\hline$(x, y)$ & \multicolumn{2}{|c|}{$(0,0)$} & \multicolumn{2}{c|}{$(-4,3.5)$} & \multicolumn{2}{c}{$(4,7)$} \\
\hline \hline$\epsilon_{\mathrm{a}, \max } / d$ & $\widehat{\rho}_{(50)}$ & $\widehat{\left|V^{e}\right|^{2}}(50)$ & $\widehat{\rho}_{(50)}$ & $\widehat{\left|V^{e}\right|^{2}}$ & $\widehat{\rho}_{(50)}$ & ${\widehat{\left|V^{e}\right|^{2}}}_{(50)}$ \\
\hline 0 & $0.26(0)$ & $0.66(-1)$ & $0.28(-2)$ & $0.42(-3)$ & $0.37(0)$ & $0.31(-1)$ \\
0.01 & $0.20(0)$ & $0.46(-1)$ & $0.33(-2)$ & $0.22(-3)$ & $0.22(0)$ & $0.49(-1)$ \\
0.03 & $0.60(0)$ & $0.13(0)$ & $0.98(-2)$ & $0.14(-2)$ & $0.37(0)$ & $0.26(-1)$ \\
0.065 & $0.23(0)$ & $0.36(-1)$ & $0.86(-1)$ & $0.54(-2)$ & $0.30(0)$ & $0.17(-1)$ \\
0.1 & $0.28(0)$ & $0.34(-2)$ & $0.27(0)$ & $0.23(-2)$ & $0.30(0)$ & $0.49(-3)$ \\
\hline
\end{tabular}

centred at the integer lattice points in the region $[-9,9] \times[-6,6]$, excluding the channel points indicated in the figure. As in the example of $\S 3$, we use a lattice constant $d=1$. The crystal structure of each realisation was perturbed by the addition to cylinder radii of random increments uniformly distributed on a specified interval $\left[-\epsilon_{\mathbf{a}, \max }, \epsilon_{\mathbf{a}, \max }\right]$. The values of $\langle\rho\rangle$ and $\left\langle\left|V^{e}\right|^{2}\right\rangle$ for TM polarisation were then estimated at each point of a square grid in the $x, y$ plane.

Using the notation of $\S 3$, Table 1 gives the values of $\widehat{\rho}_{(50)}$ and $\widehat{\left.V^{e}\right|^{2}}$ (50) at selected points in the channel, in the crystal and outside the crystal, for a range of levels of perturbation $\epsilon_{\mathbf{a} \text {,max }}$. The wavelength used $(\lambda / d=3.3)$ lies in the band gap for the crystal. High values of $\widehat{\left.V^{e}\right|^{2}}{ }_{(50)}$ in the channel, together with low values in the matrix, indicate strong guiding in the weak randomisation cases. With strong randomisation $\left(\epsilon_{\mathbf{a}, \max } / d=0.1\right)$ the values of $\hat{\rho}_{(50)}$ are all reasonably close to the free space value $(0.25)$ - the structure's influence on local radiation dynamics is relatively minor. Further, with strong randomisation, the relatively low values of $\widehat{\left|V^{e}\right|^{2}}{ }_{(50)}$ at points at the channel exit and in the channel, but beyond its first segment, indicate effective closure of the channel.

Figure 4 gives contour plots of $\widehat{\rho}_{(50)}$ and $\widehat{\left|V^{e}\right|^{2}}{ }_{(50)}$. The cylinder positions of the ideal structure are indicated by the circles in the plot. For $\epsilon_{\mathbf{a}, \max } / d=0.01,0.03$ the figure demonstrates the suppression of propagation within the structure except in the waveguide, indicating that the crystal possesses substantial guiding properties. However, for $\epsilon_{\mathrm{a}, \max } / d=0.065$ both the LDOS and the Green's function plots suggest that there is substantial leakage from the guiding channel. At the $\epsilon_{\mathbf{a}, \max } / d=0.1$ level this leakage is so severe that guiding has effectively ceased. These observations are consistent with the data in Table 1. Data such as that presented in Table 1 and Fig. 4 may provide substantial 


$$
\epsilon_{\mathbf{a}, \max } / d=0.01
$$
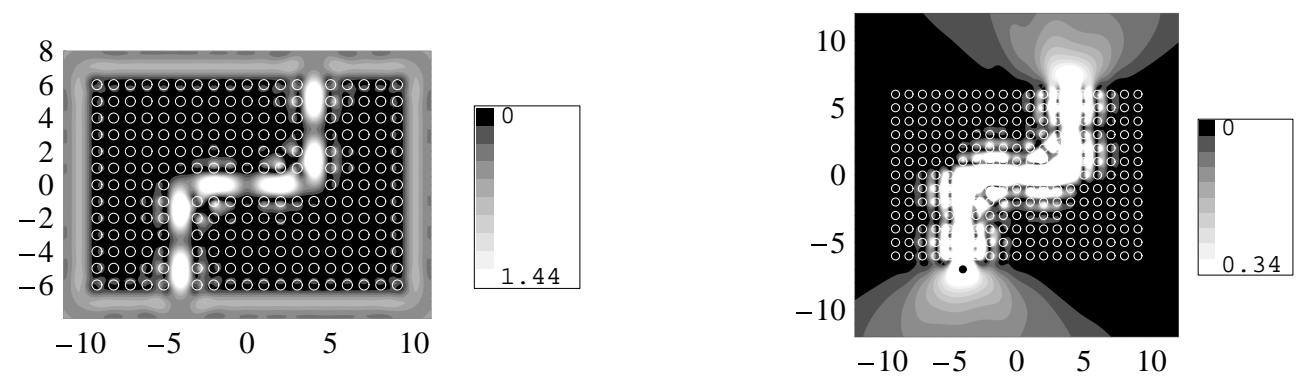

$$
\epsilon_{\mathbf{a}, \max } / d=0.03
$$
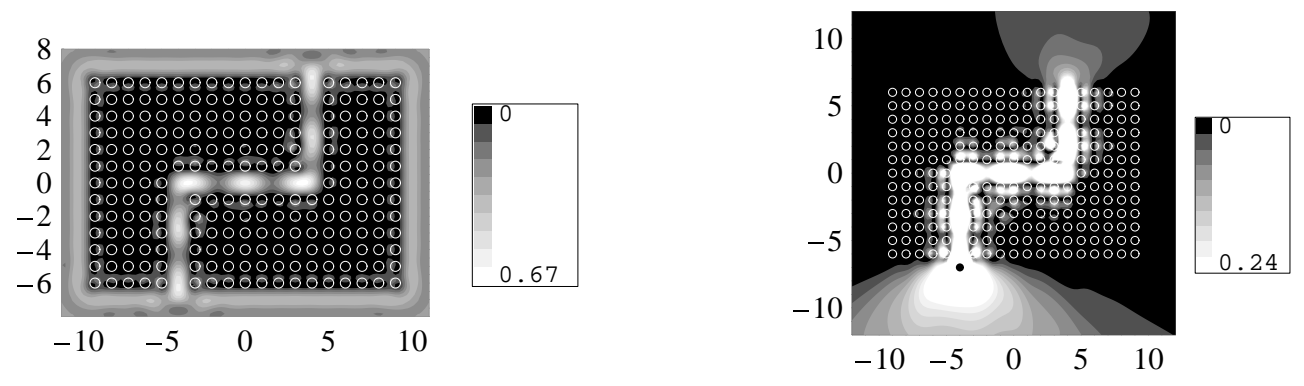

$$
\epsilon_{\mathbf{a}, \max } / d=0.065
$$
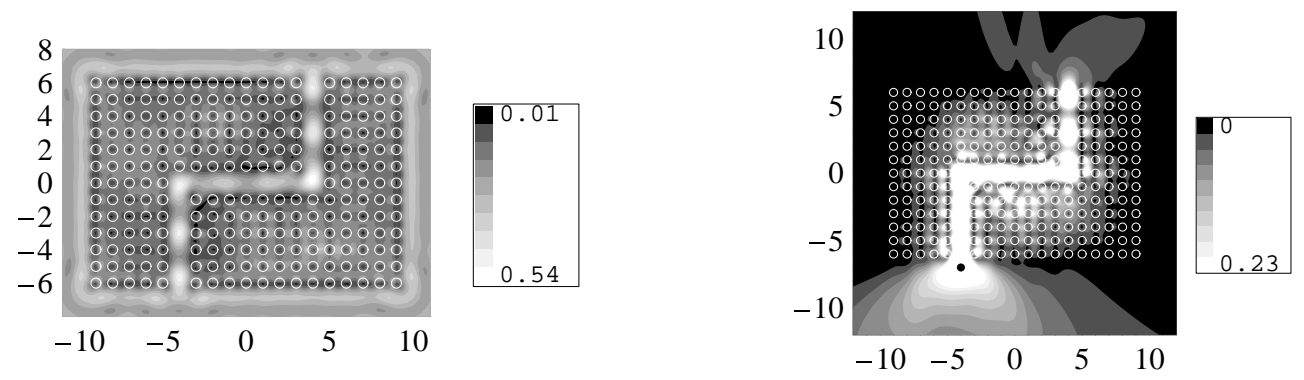

$\epsilon_{\mathbf{a}, \max } / d=0.1$
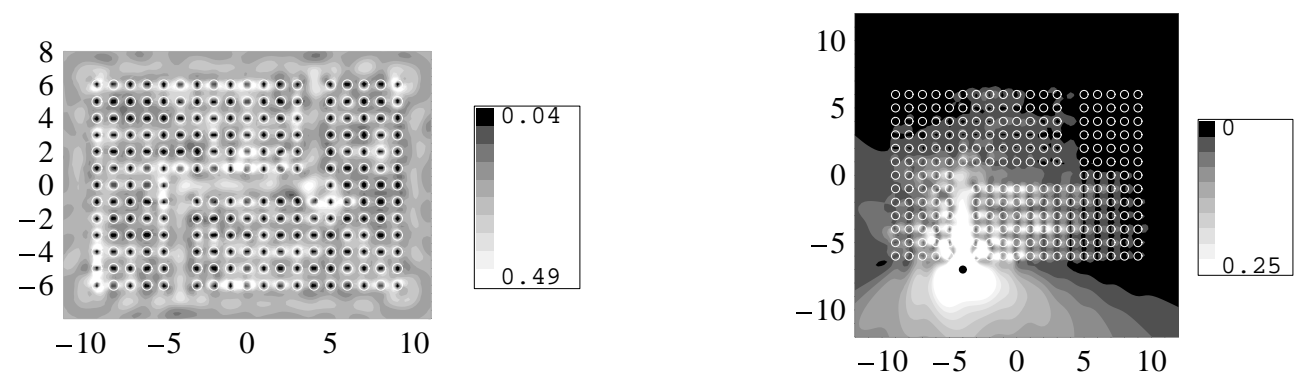

Fig. 4. Contour plots of $\widehat{\rho}_{(50)}$ (left) and ${\widehat{\left|V^{e}\right|^{2}}}_{(50)}$ (right) for TM polarisation for a waveguide $\left(N_{c}=226\right)$ composed of infinite circular cylinders with increasingly disordered radii. The crystal's design parameters and the wavelength used are the same as those of Fig. 3. In particular, the design radius is $0.3 d$ units. 
benefits in the manufacture of photonic crystal waveguides.

\section{References}

[1] J. Hecht, A. Zajac, Optics, Addison-Wesley Publishing Co., Reading, Massachusetts, 1987.

[2] J. Hecht, Holes in photonic crystal fibers open new possibilities, Laser Focus World 37 (5) (2001) 207-216.

[3] H. Jones-Bey, Optical integrated circuits - photonic bandgap channels waves propagating at metal surfaces, Laser Focus World 37 (6) (2001) 22-26.

[4] C. M. Soukoulis (Ed.), Photonic Band Gap Materials, Vol. 315 of NATO ASI Series, Kluwer Academic Publishers, Dordrecht, the Netherlands, 1996.

[5] A. Asatryan, K. Busch, R. C. McPhedran, L. C. Botten, C. M. de Sterke, N. A. Nicorovici, Two-dimensional Green's function and local density of states in photonic crystals consisting of a finite number of cylinders of infinite length, Phys. Rev. E 63 (4, art. no. 046612) (2001) 46612-1-46612-4.

[6] A. Asatryan, S. Fabre, K. Busch, R. C. McPhedran, L. C. Botten, C. M. de Sterke, N. A. Nicorovici, Two-dimensional local density of states in twodimensional photonic crystals, Optics Express 8 (3) (2001) 191-196.

[7] L. C. Botten, R. C. McPhedran, N. A. Nicorovici, A. A. Asatryan, C. M. de Sterke, P. A. Robinson, K. Busch, G. H. Smith, T. N. Langtry, Rayleigh multipole methods for photonic crystal calculations, in: PIER Special Issue on Electromagnetic Applications of Photonic Band Gap Materials and Structures, (in press).

[8] S. John, K. Busch, Photonic bandgap formation and tunability in certain selforganizing systems, Journal of Lightwave Technology 17 (11) (1999) 1931-1943.

[9] A. Moroz, Minima and maxima of the local density of states in periodic systems, Europhys. Lett. 46 (1999) 419-424.

[10] R. Balian, C. Bloch, Distribution of Eigenfrequencies for the wave equation in a finite domain II. Electromagnetic field. Riemannian spaces, Annals of Physics 64 (1971) 271-307.

[11] M. H. Kalos, P. A. Whitlock, Monte Carlo Methods, John Wiley and Sons, New York, 1986.

[12] G. S. Fishman, Multiplicative congruential random number generators with modulus $2^{b}$ : an exhaustive analysis for $b=32$ and a partial analysis for $b=48$, Math. Comp. 54 (189) (1990) 331-344.

[13] B. L. Fox, Algorithm 647: implementation and relative efficiency of quasirandom sequence generators, ACM Transactions on Mathematical Software 12 (4) (1986) 362-376. 